\title{
Probing the Spatial Structure of a Molecular Attosecond Electron Wave Packet Using Shaped Recollision Trajectories
}

\author{
Hiromichi Niikura, ${ }^{1,2,3, *}$ Hans Jakob Wörner, ${ }^{1,4}$ D. M. Villeneuve, ${ }^{1}$ and P. B. Corkum ${ }^{1,5}$ \\ ${ }^{1}$ National Research Council of Canada, 100 Sussex Drive, Ottawa, Ontario, Canada K1A0R6 \\ ${ }^{2}$ PRESTO, Japan Science and Technology Agency, Sanbancho building, 5-Sanbancho, Chiyodaku, Tokyo 102-0075, Japan \\ ${ }^{3}$ Department of Applied Physics, Waseda University, Okubo3-4-1, Shinjyuku, Tokyo 169-8555, Japan \\ ${ }^{4}$ Laboratorium für physikalische Chemie, ETH Zürich, Wolfgang-Pauli-Strasse 10, 8093 Zürich, Switzerland \\ ${ }^{5}$ Department of Physics, University of Ottawa, 150 Louis Pasteur, Ottawa, Ontario, Canada K1N 6N5
}

(Received 29 November 2010; published 22 August 2011)

\begin{abstract}
Using orthogonally polarized $800 \mathrm{~nm}$ and $400 \mathrm{~nm}$ laser pulses, we have generated high harmonics in ethane $\left(\mathrm{C}_{2} \mathrm{H}_{6}\right)$. We observe that the intensity of each harmonic order modulates with the attosecond delay between the two laser fields. The modulation period of the low even harmonics is twice that of the period of modulation of the other harmonics. By comparing with theoretical calculation, we show that the double periodicity is a result of the electron wave packet motion in the valence shell of $\mathrm{C}_{2} \mathrm{H}_{6}$ on the attosecond time-scale. Our method is a general approach to measuring internal electron dynamics which does not require molecular alignment, making it applicable to more complex molecules than previous approaches.
\end{abstract}

DOI: 10.1103/PhysRevLett.107.093004

PACS numbers: $33.80 . \mathrm{Rv}, 42.50 . \mathrm{Hz}$

Attosecond electron wave packets in the valence shell of molecules are an unexplored but common feature of photochemical reactions, photoionization and charge transfer in molecules [1-5]. When intense, infrared laser pulses interact with a molecule whose cation's electronic states lie close together, tunnel ionization may prepare several of them [6-8]. In 2009, it was suggested that this process can create an electron wave packet or electron-hole dynamics in the valence shell of a molecule which was inferred from high-harmonic generation in aligned $\mathrm{CO}_{2}$ molecules [6]. However, there are several difficulties in applying this method directly to other molecules. First, although an intensity minimum was observed in the high-harmonic spectra and attributed to the interference between the emission from two ionization channels, such interference minima are insensitive to the electronic coherence between the two states of the cation and would also appear if emission was occurring from two independent species [9]. Second, because one linearly polarized laser pulse is responsible for both tunnel ionization and the electron recollision processes, the spatial structure of the internal and continuum electron wave packets are influenced by angle of the molecular alignment with respect to the field direction. It is thus difficult to disentangle ionization and recombination which can only be achieved through extensive theoretical calculations. Third, it is difficult to trace spatial properties of the electron wave packet as a function of time.

Using $\mathrm{C}_{2} \mathrm{H}_{6}$, we demonstrate that the dynamical changes in the spatial structure of an electron wave packet in the valence shell is observed with attosecond time-resolution. To measure the dynamics, we employ a two-color laser field approach with unaligned molecules [10,11]. This approach allows us to identify the spatial structure or transient symmetry of an electron wave packet in a molecule through the high-harmonic generation process. The symmetry of the wave packet is mapped onto the polarization of the high-harmonic spectra which are measured as a function of the relative delay between the two laser pulses. It has advantages over the alignment method employed in Ref. [6] in terms of measuring electron wave packet motion. First, the continuum and the bound valence electron wave packets produced at the time of ionization are essentially unchanged as a function of the delay between the two laser pulses. Ionization will select the subset of molecules that is aligned along the most favorable direction. The continuum wave packet will initially move along the direction given by the field. This permits disentangling of the ionization from the probing recollision process, which simplifies the analysis. Second, we can observe directly how the bound wave packet structure as seen by the continuum electron changes in time. This becomes possible because the continuum wave packet recombines with the parent ion from different angles, controlled by the delay between the two colors. The instantaneous electronic structure is mapped into the polarization direction of harmonics as a function of the recollision angle.

Figure 1(a) shows the concept of the measurement. We combine an $800 \mathrm{~nm}$ laser pulse with its second harmonic spatially and temporally. The polarization direction of the $800 \mathrm{~nm}$ field is orthogonal to that of the second harmonic. At the peak of the combined laser fields, tunnel ionization of molecules forms a correlated electron wave packet pair. One moves in the valence shell of the molecule and the other moves in the ionization continuum. With unaligned molecules, if tunnel ionization probability is directional to a specific range of angles relative to the molecular principal axis, the ionized molecular ensemble is restricted to 

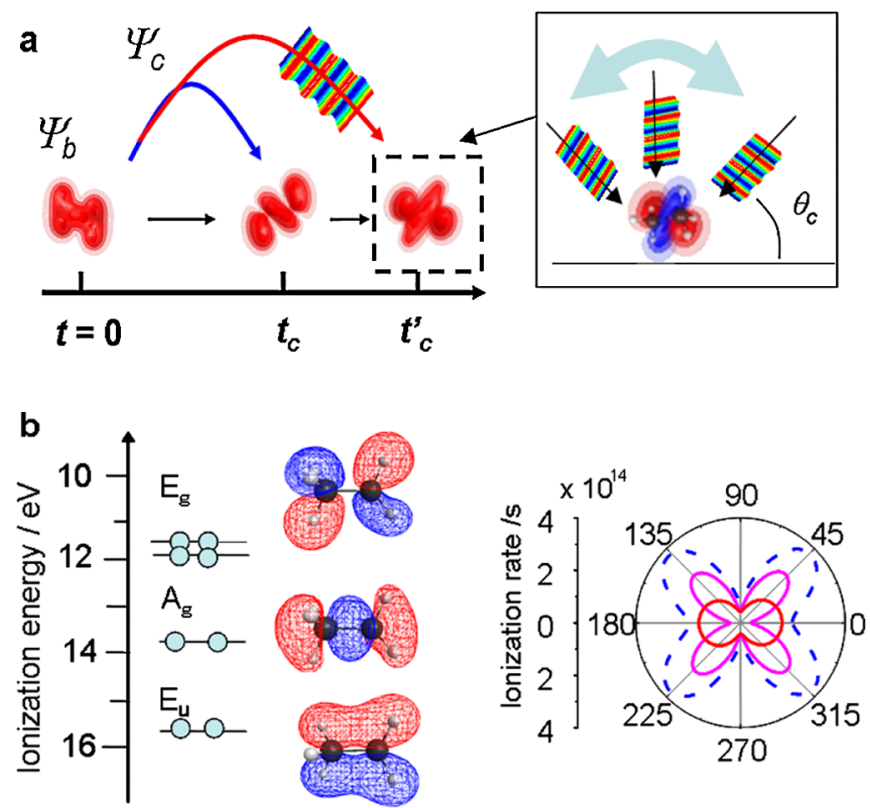

FIG. 1 (color online). (a) Tunnel ionization creates wave packet pairs in the ionization continuum $\left(\Psi_{c}\right)$ and in the valence shell $\left(\Psi_{b}\right)$. Upon recombination of two wave packets, high harmonics are emitted. At each recollision time, we scan the recollision angle $\left(\theta_{c}\right)$ with respect to the molecule by scanning the phase delay between the 800 and $400 \mathrm{~nm}$ laser fields. The time of recollision is determined by the observed photon energy of the high-harmonic emission. We read the structure of the valence electron wave packet from the relationship between the polarization angle of the high harmonics and the recollision angle (see text). (b) (left) Schematic diagram of the calculated, selected valence orbitals of $\mathrm{C}_{2} \mathrm{H}_{6}$. (right) The calculated angular distribution of the tunnel ionization probability for the most loosely bound orbital ( $E_{g}$ symmetry, magenta line) and the second most loosely bound orbital ( $A_{g}$ symmetry, red line) of $\mathrm{C}_{2} \mathrm{H}_{6}$ at the laser intensity of $2.0 \times 10^{14} \mathrm{~W} / \mathrm{cm}^{2}$. We show only one of the two degenerate $E_{g}$ orbitals. The dotted line is the incoherent sum of these two lines. The ionization probability of the other states is negligible.

that specific angular range [10]. This property determines molecular alignment through the field direction at the time of ionization. Because the amplitude and the direction of the sum of the two laser fields change continuously through one optical cycle, the continuum electron trajectory is controlled by the field and angle relative the initial direction into which ionization took place. Within less than one optical period, the continuum electron recombines with the bound electron wave packet, leading to high-harmonic emission. The direction of the induced dipole moment, i.e., the polarization angle of harmonics $\left(\phi_{\mathrm{HHG}}\right)$ depends on the recollision angle, $\theta_{c}$, and the structure and symmetry of the valence state wave packet. The recollision angle can be controlled by changing the delay between the two laser fields. The variation of $\phi_{\mathrm{HHG}}$ with $\theta_{c}$ depends characteristically on the alignment and the spatial symmetry of the valence state electron wave packet [10]. Therefore, if dynamical motion occurs in the valence state, then the dependence of $\phi_{\mathrm{HHG}}$ on $\theta_{c}$ tracks its motion. Because there is a one-to-one correspondence between photon energy and the transit time $t_{c}$ of the continuum electron, the dynamical motion is mapped on the photon energy when we choose an appropriate phase matching condition to isolate the short trajectories [12].

We obtain the relative value of $\phi_{\mathrm{HHG}}$ from the measured high-harmonic generation spectra by comparing the intensity ratio between the adjacent odd and even harmonics $[10,11]$. This is based on the fact that even (odd) harmonics are polarized parallel to the $400 \mathrm{~nm}(800 \mathrm{~nm})$ polarization axis, respectively. We utilize $\mathrm{H}_{2}$ as a reference molecule to obtain $\theta_{c}$ as a function of the harmonic number. With unaligned molecules, $\phi_{\mathrm{HHG}}$ of $\mathrm{H}_{2}$ is approximately equal to $\theta_{c}$ because the angular dependence of the tunnel ionization probability is close to spherical [13]. Therefore, comparison of $\phi_{\mathrm{HHG}}$ of $\mathrm{H}_{2}$ with that of $\mathrm{C}_{2} \mathrm{H}_{6}$ provides the polarization angle changes as a function of the recollision angle.

In Fig. 1(b), we present the structure of the valence orbitals of $\mathrm{C}_{2} \mathrm{H}_{6}$ obtained by ab initio calculation and the experimental vertical ionization energies [14,15]. We also plot the calculated angular dependence of the tunnel ionization probability for the $E_{g}$ and $A_{g}$ orbitals at the total laser intensity of $2.0 \times 10^{14} \mathrm{~W} / \mathrm{cm}^{2}$, respectively, obtained by using the approach described in Ref. [16]. The dotted line shows the incoherent sum of both tunnel ionization probabilities. It has maximum values around angles of $\sim 45 \pm 90$ and 225 degrees. The internal electron wave packet is prepared by simultaneous ionization from the $E_{g}$ and $A_{g}$ orbitals. The electron wave function at the time of recombination is approximately given by

$$
\begin{aligned}
\Psi(t) & \propto \Psi_{b} \Psi_{c}, \\
\Psi_{b} & =a(1) \Psi_{b}\left(E_{g}\right)+a(2) \Psi_{b}\left(A_{g}\right) \exp (-i \varphi(t))
\end{aligned}
$$

where $a(1)$ and $a(2)$ are coefficients, $\Psi_{c}$ is the continuum electron wave packet and $\varphi(t)$ is the phase difference between the two states. $\Psi_{b}\left(E_{g}\right)$ and $\Psi_{b}\left(A_{g}\right)$ are the wave functions of the electron hole or, equivalently, the Dyson orbital of each ionization channel, where one electron is removed from the most and the second most loosely bound state of $\mathrm{C}_{2} \mathrm{H}_{6}$, respectively. We neglect the effects of electron correlation on orbital relaxation. The coherent superposition between the two wave functions generates dynamical motion of the electron vacancy, i.e., hole dynamics or electron wave packet motion in the molecule. Phase matching requires that the continuum electron returns to its initial bound state, forcing the electron to recombine to a new location and a changed hole distribution.

We generate an intense $800 \mathrm{~nm}$ laser pulse of $35 \mathrm{fs}$ pulse duration from a Ti:sapphire laser system (KMlabs). The frequency of the $800 \mathrm{~nm}$ pulse is doubled in a $300 \mu \mathrm{m}$ 
thick $\beta-\mathrm{BaB}_{2} \mathrm{O}_{4}$ crystal. Both laser pulses coaxially traverse a $0.65 \mathrm{~mm}$ calcite plate. By rotating the calcite plate around an axis perpendicular to the propagation direction to control the optical path length, we adjust the phase delay between the two laser pulses [10]. We focus the laser pulses downstream of a pulsed gas jet with a $50 \mathrm{~cm}$ focal length spherical mirror. The generated harmonics are dispersed by a flat-field grating (Hitachi, 001-226). The dispersed spectrum is imaged onto a two-dimensional microchannel plate and a phosphor screen. We capture the image by a CCD camera and transfer it to a computer. We integrate the signal counts in the direction perpendicular to the dispersed axis.

In Fig. 2, we show the measured high-harmonic emission spectra of $\mathrm{C}_{2} \mathrm{H}_{6}$ as a function of the relative delay between the two laser pulses at the total laser intensity of $2.0 \times 10^{14} \mathrm{~W} / \mathrm{cm}^{2}$. For reference, we measure the spectra of high harmonics generated from $\mathrm{H}_{2}$ and $\mathrm{N}_{2}$ as well using the same laser field (not shown). Except for 14th and 16th harmonics, the intensity of each harmonic emission of $\mathrm{C}_{2} \mathrm{H}_{6}$ modulates with half of the $400 \mathrm{~nm}$ optical period or $0.66 \mathrm{fs}$. For harmonics 14 and 16, two intensity peaks are observed within every half-period of the $400 \mathrm{~nm}$ field. One peak appears at approximately the same delay as the adjacent odd harmonic $(\sim 0.33$ and $\sim 0.99$ fs $)$, while the other peak appears halfway between them $(\sim 0.66$ and $\sim 1.33 \mathrm{fs}$ ). These two peaks coalesce to one peak in the harmonic orders above the 18th. In the case of $\mathrm{H}_{2}$ and $\mathrm{N}_{2}$, all harmonics are modulated with half of the $400 \mathrm{~nm}$ optical period.

In order to interpret these results, we show $\phi_{\mathrm{HHG}}$ measured in $\mathrm{H}_{2}$ and $\mathrm{C}_{2} \mathrm{H}_{6}$ in Figs. 3(a) and 3(b). One $\phi_{\text {HHG }}$ peak is found in $\mathrm{H}_{2}$ while two $\phi_{\text {HHG }}$ peaks are found in $\mathrm{C}_{2} \mathrm{H}_{6}$ at every half of the $400 \mathrm{~nm}$ period. In the range

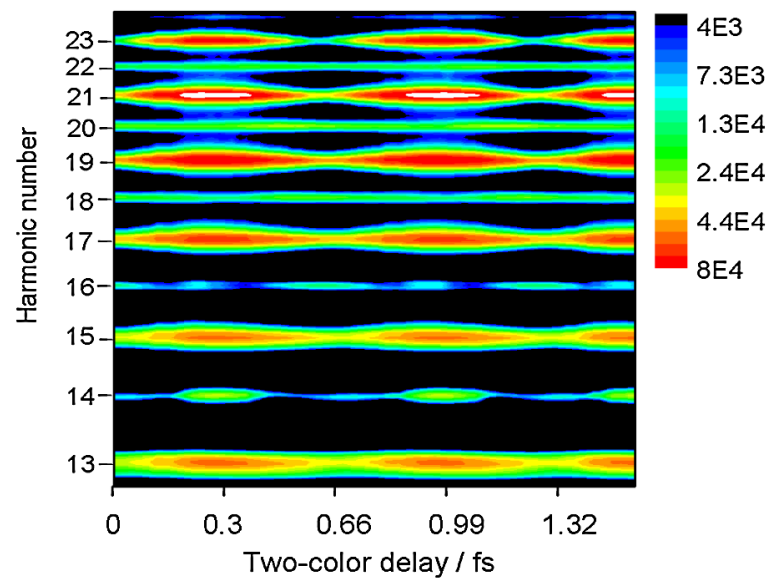

FIG. 2 (color online). High-harmonic generation spectra from $\mathrm{C}_{2} \mathrm{H}_{6}$ as a function of the delay between the two laser pulses. We choose the zero-delay arbitrarily. The total harmonic intensity modulates with the period of $0.66 \mathrm{fs}$ while the intensity of 14th and 16th harmonics modulates with the period of $0.33 \mathrm{fs}$. from harmonics 13 to 15 at the delay of $\sim 0.33$ and $\sim 1.00 \mathrm{fs}, \phi_{\mathrm{HHG}}$ of $\mathrm{C}_{2} \mathrm{H}_{6}$ is approximately proportional to $\phi_{\mathrm{HHG}}\left(=\theta_{c}\right)$ of $\mathrm{H}_{2}$. This indicates that the dipole moment responsible for high-harmonic emission is induced approximately parallel to the recollision angle. This is consistent with a $\sigma$-like symmetry of the molecular orbital aligned parallel to the recollision angle [10]. In the range from the 16 th to the 21 st harmonic at the delay of $\sim 0,0.66$ and $1.33 \mathrm{fs}, \phi_{\mathrm{HHG}}$ of $\mathrm{C}_{2} \mathrm{H}_{6}$ has a peak at $\sim 45$ degrees while $\phi_{\mathrm{HHG}}$ of $\mathrm{H}_{2}$ is $\sim 0$ degree. This indicates that the dipole moment is induced at an angle different from the recollision direction. Thus, the spatial structure of $\Psi_{b}$ must be different at the recollision times corresponding to the low harmonics ( $\sim 13$ th $)$ and the high harmonics ( $\sim 18$ th). To exhibit the relationship of the recollision time and the harmonic number, we plot the recollision time obtained by classical electron trajectory calculation [17] in the right axis of Fig. 3. The harmonic orders 13 and 18 correspond to a recollision time of $\sim 0.8$ and $1.2 \mathrm{fs}$, respectively.

We have repeated the same measurement at four different driving laser intensities in the range from $1.1 \times 10^{14} \mathrm{~W} / \mathrm{cm}^{2}$ to $1.7 \times 10^{14} \mathrm{~W} / \mathrm{cm}^{2}$. From the observed spectra, we obtain the $\phi_{\mathrm{HHG}}$ distribution for each laser intensity as shown in the online material [18]. The highest harmonic number where the double intensity peak appears, shifts from the 16th to the 14th order and the $\phi_{\text {HHG }}$ distribution patterns shift to lower harmonic numbers as the laser intensity decreases. Thus, the possibilities of interference specific to the molecular structure and the electron wave function can be excluded. This is because in the case of structural interference, the characteristic intensity minimum must be independent of the driving laser intensity $[6,19]$. When we convert the harmonic number to the

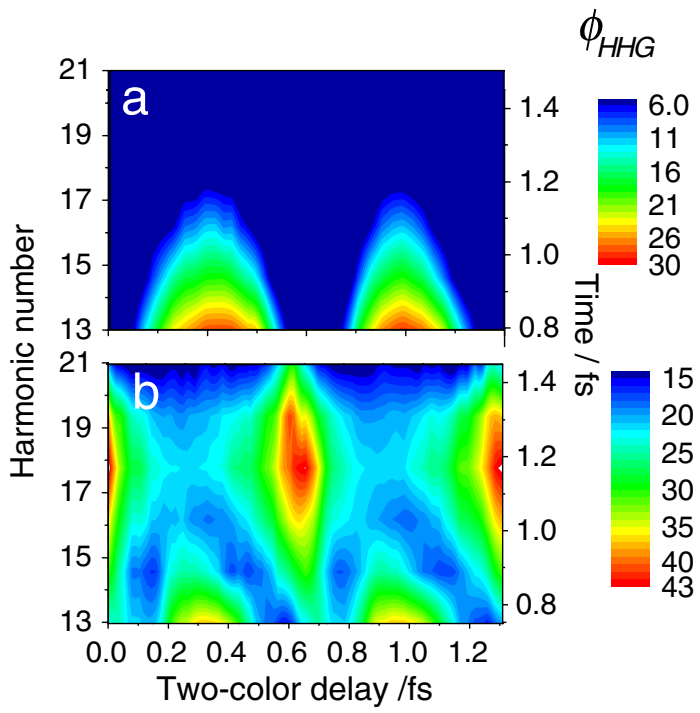

FIG. 3 (color online). $\phi_{\mathrm{HHG}}$ (in degrees) as a function of the delay between the two laser pulses for (a) $\mathrm{H}_{2}$ and (b) $\mathrm{C}_{2} \mathrm{H}_{6}$. We interpolate $\phi_{\mathrm{HHG}}$ along the two axes. The right axis shows the recollision time which is calculated from the harmonic number. 
recollision time at each laser intensity using classical electron trajectory calculations, we find that the $\phi_{\mathrm{HHG}}$ peak appearing at the delay of $0, \sim 0.66$, and $\sim 1.33$ fs stays at the same recollision time of $\sim 1.2 \mathrm{fs}$ regardless of the laser intensity. Therefore, the experimental result is consistent with the prediction that the tunnel ionization prepares the electron wave packet motion in the molecule [6].

In what follows, we identify the dynamics of $\Psi_{b}$ by simulating $\phi_{\mathrm{HHG}}$ as a function of the high-harmonic number and the two-color delay using a semiclassical, strong-field approximation [17]. First, we calculate electron trajectories and $\theta_{c}$ under the two-color laser fields. Then we calculate $\phi_{\mathrm{HHG}}$ from the high-harmonic intensity induced parallel to the $800 \mathrm{~nm}$ axis and the $400 \mathrm{~nm}$ axis [10]. We use Eq. (1) to generate the bound state wave packet, $\Psi_{b}$. We neglect the Stark shift difference between the two electronic states and nonadiabatic population transfer because the transition dipole moment between them is zero since they have the same parity. In this case, the phase evolution in Eq. (1) is given by

$$
\varphi(t)=\Delta E / \hbar \tau_{c}+\varphi(0),
$$

where $\Delta E$ is the energy separation between the $E_{g}$ and $A_{g}$ states, and $\varphi(0)$ is the initial phase shift which is determined at the time of tunnel ionization. For characterizing the wave packet, $\Delta E$ and $\varphi(0)$ are the parameters to be adjusted. We rely on $\Delta E \sim 2 \mathrm{eV}$ from a result of ab-initio calculations and measured photoelectron spectra [14]. At fixed $\Delta E=$ $2 \mathrm{eV}$, we adjust $\varphi(0)$ over a $2 \pi$ range to fit the observed $\phi_{\mathrm{HHG}}$ to the calculated $\phi_{\mathrm{HHG}}$ distribution. We find a good agreement between theory and experiment for $\varphi(0)=$ $0.4 \pi$. In Figs. 4(a) and 4(b), we show the calculated $\phi_{\mathrm{HHG}}$ at $\varphi(0)=-0.4 \pi$ and $\varphi(0)=0.4 \pi$, respectively. We show the case $\varphi(0)=-0.4 \pi$ to demonstrate how the $\phi_{\mathrm{HHG}}$ distribution is sensitive to the initial phase difference. As we change $\varphi(0)$ from $-0.4 \pi$ to $0.4 \pi$, the two $\phi_{\mathrm{HHG}}$ peaks shift from small to large recollision time. In order to show the relationship between $\phi_{\mathrm{HHG}}$ and $\left|\Psi_{b}\right|^{2}$ at each recollision time, we present the calculated $\left|\Psi_{b}\right|^{2}$ at selected recollision times of $t_{c}=0.8,1.0$, and $1.2 \mathrm{fs}$ for the case of $\varphi(0)=0.4 \pi$ in Fig. 4.

To confirm that the double periodicity of $\phi_{\mathrm{HHG}}$ is due to the dynamics, we calculate $\phi_{\mathrm{HHG}}$ as a function of the harmonic order and the delay using only one of the $E_{g}$ or $A_{g}$ states. The sum of $\phi_{\mathrm{HHG}}$ peaks calculated from only the $E_{g}$ or $A_{g}$ state does not agree with the observed $\phi_{\mathrm{HHG}}$ distribution. We also calculate the angular distribution of the tunnel ionization probability from the coherent superposition of the two states for $\varphi(0)$ of $0.4 \pi$. The distribution is very close to the result obtained for an incoherent sum.

Before concluding, we present possibilities how the phase shift between two states, $\varphi(0)$, can be caused at the time of tunnel ionization. The recolliding electron wave packet $\left(\Psi_{c}\right)$ generated from different electronic states must

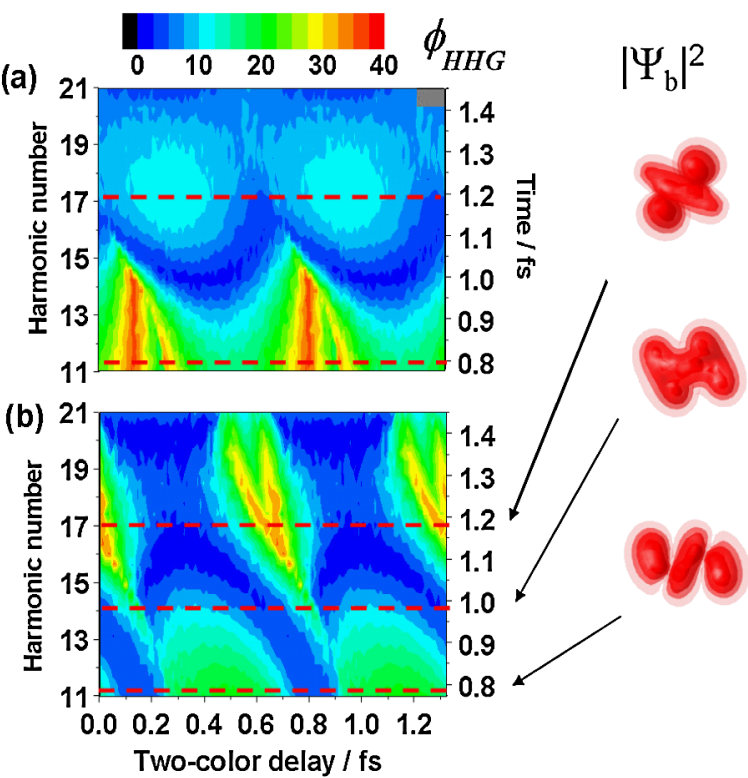

FIG. 4 (color online). Calculated $\phi_{\mathrm{HHG}}$ (in degrees) as a function of the harmonic order and the phase delay for an initial phase $[\phi(0)]$ of (a) $-0.4 \pi$ and (b) $0.4 \pi$. We use the energy difference between the most and the second most loosely bound orbitals of $\mathrm{C}_{2} \mathrm{H}_{6}, \Delta E=2.0 \mathrm{eV}$. The right panel shows the calculated $\left|\Psi_{b}\right|^{2}$ at selected recollision time.

constructively interfere with each other in order to lead to efficient high-harmonic generation. This requirement may select ensembles of the bound state electron wave functions with a particular phase difference so that the $\Psi_{c}$ can constructively interfere. Other possibilities include the effect of the laser fields which can change the timeevolution of the wave function, or the effect of different electrostatic potentials of the two channels on the outgoing and recombining electrons.

In summary, we have demonstrated that dynamical motion of the valence electron wave packet is mapped on the high-harmonic emission spectra generated by a two-color laser field. By fitting the calculated observed polarization map to the observed one, the initial phase difference is obtained. For further applications, our approach can be extended to measuring dynamics associated with changes in the photo-recombination dipole moment such as internal electron motion induced by intense laser fields, core-level ionization, chemical reactions, external atom migration or molecular isomerization [20,21]. If we use an infrared laser pulse such as $2000 \mathrm{~nm}$ and its second harmonic, then we can increase $\theta_{c}$ over $90^{\circ}$ and extend the harmonic number because the continuum electron achieves a higher recollision energy. If the amplitude of the dipole moment vector as well as $\phi_{\mathrm{HHG}}$ can be obtained, then we expect to reconstruct the spatial structure of the electronic wave packet as it changes by combining with molecular alignment and orientation techniques [22].

H. J.W. acknowledges support through SNSF Grant No. PP00P2_128274. 
*Corresponding author. niikura@waseda.jp

[1] F. Krausz and M. Ivanov, Rev. Mod. Phys. 81, 163 (2009).

[2] F. Remacle and R. D. Levine, Z. Phys. Chem. 221, 647 (2007).

[3] G. Sansone et al., Nature (London) 465, 763 (2010).

[4] E. Goulielmakis et al., Nature (London) 466, 739 (2010).

[5] H. J. Wörner et al., Phys. Rev. Lett. 104, 233904 (2010).

[6] O. Smirnova et al., Nature (London) 460, 972 (2009).

[7] S. Haessler et al., Nature Phys. 6, 200 (2010).

[8] H. Akagi et al., Science 325, 1364 (2009).

[9] T. Kanai, E. Takahashi, Y. Nabekawa, and K. Midorikawa, Phys. Rev. Lett. 98, 153904 (2007).

[10] H. Niikura, N. Dudovich, D. Villeneuve, and P. B. Corkum, Phys. Rev. Lett. 105, 053003 (2010).

[11] D. Shafir et al., Nature Phys. 5, 412 (2009).

[12] Y. Mairesse et al., Science 302, 1540 (2003).
[13] A. Staudte et al., Phys. Rev. Lett. 102, 033004 (2009).

[14] A. Richartz, R. J. Buenker, P. J. Bruna, and S.D. Peyerimhoff, Mol. Phys. 33, 1345 (1977).

[15] M. W. Schmidt et al., J. Comput. Chem. 14, 1347 (1993).

[16] J. Muth-Böhm, A. Becker, and F. Faisal, Phys. Rev. Lett. 85, 2280 (2000).

[17] P. Corkum, Phys. Rev. Lett. 71, 1994 (1993).

[18] See Supplemental Material at http://link.aps.org/ supplemental/10.1103/PhysRevLett.107.093004 for the intensity dependent spectra and analysis.

[19] H. J. Wörner, H. Niikura, J. B. Bertrand, P. B. Corkum and D. M. Villeneuve, Phys. Rev. Lett. 102, 103901 (2009).

[20] H. J. Wörner et al., Nature (London) 466, 604 (2010).

[21] W. Li et al., Proc. Natl. Acad. Sci. U.S.A. 107, 20219 (2010).

[22] J. Itatani et al., Nature (London) 432, 867 (2004). 